P96 (continued)

Outcome Measures and Analysis: To determine individual $\mathrm{FV}$ weights a minimum of 3 individual pieces of each FV on the salad bar were weighed and averaged. Average weights were multiplied by number of pieces taken by each student. To determine pan weights, each pan of specific FV was weighed before and after students went through lunch line. Difference between pre and post weights was divided by number of students selecting a particular FV to derive portion weight. To validate these 2 methods, actual salad bar portions were also weighed. Estimations using individual FV and pan weights were then compared to each other and with actual weighed portions using paired t-tests.

Results: FV portions measured from 47 lunch trays. Mean weighed salad bar portions $=148 \mathrm{~g}$, mean portion from individual $\mathrm{FV}$ weights $=142 \mathrm{~g}$, mean portion from pan weights $=131 \mathrm{~g}$. Differences were not significant. Although individual FV and pan weights both estimated portion weights similarly to actual portion weights, pan weight method tended to underestimate portion weights.

Conclusions and Implications: Variability in children's self-service salad bar portions chosen presents challenges for portion size determination. Findings support use of either individual FV or pan weights as valid methods to estimate student portions.

Funding: NIFA.

\section{P97 Assessment of Food Waste Knowledge, Attitudes, and Behaviors of University Students}

Lynn Luecke, lcluecke@stkate.edu, St. Catherine University, 2004 Randolph Avenue, St. Paul, MN 55105; T. Burgess-Champoux, PhD, RD, LDN

Objective: To determine university students' knowledge, attitudes, and behaviors about food waste and evaluate the impact of a text message educational intervention on individual food waste.

Design, Setting and Participants: In university settings across the United States, about 3.6 million tons of food is wasted annually. A growing body of research has been conducted in university settings, yet additional work is necessary to understand food waste perceptions and behaviors of young adults. A convenience sample of undergraduate students with meal plans living on campus at a private university for women in Minnesota were recruited during fall $2014(\mathrm{n}=55)$. Food waste knowledge, behaviors and individual plate waste was measured at baseline and post-intervention. Social Cognitive Theory provided the framework for survey questions. Educational text messages delivered over 4 weeks focused on four food waste themes identified from the baseline survey results.

Outcome Measures and Analysis: Intervention impact was measured using pre-post knowledge, attitudes and behaviors pertaining to food waste and individual food waste measurements.
Results: Baseline survey responses suggest that participants often keep leftovers (80.5\%) and follow use-by dates on packages (70.7\%). Environmental sustainability is very important to participants $(70.7 \%)$ yet only $51.2 \%$ reported an excellent understanding of environmental sustainability. Student perceptions of the educational text messages were mixed. Pre/post food waste differences were not significantly different $(n=32 ; \mathrm{p}=0.42)$. Data analysis is ongoing.

Conclusions and Implications: Food waste and sustainability are important issues to university students. Food is to be shared and valued, not wasted thoughtlessly. Future research should include a larger, more diverse sample in a variety of university foodservice setting.

Funding: None.

\section{P98 Improving Food Infrastructure to Create Health Equity: Community Voices on a Regional Food Hub Model for Brooklyn, NY}

Heewon Gray, PhD, RD, hl2001@tc.columbia.edu, Teachers College, Columbia University, 525 West 120th Street, Box137, New York, NY 10027; H. Bhana, MS; R. Paul, MS, RD; P. Koch, EdD, RD; I. Contento, PhD

Objective: One solution for a sustainable food system is a "food hub model" where food from local farms is aggregated and then distributed to stores, farmers markets, food pantries, etc. This study, within a low-income urban setting, examined residents' food purchases and demands for local products, and community leaders' opinions about food hubs.

Design, Setting and Participants: The research area was four Community Districts in Brooklyn, New York. Residents, organizational leaders (OLs), and urban farmers participated in a mixed-method, cross-sectional study $(\mathrm{n}=141)$.

Outcome Measures and Analysis: Community residents completed a 21-item survey $(\mathrm{n}=80)$ and participated in 5 focus groups $(n=50)$ which collected data on food purchasing patterns and demands. Three OLs answered a 10-item regional food environment questionnaire. Four OLs and 4 urban farmers participated in semi-structured interviews asking opinions on a food hub model. Quantitative data were analyzed using descriptive statistics. Qualitative data were transcribed verbatim and analyzed with in-vivo coding to identify themes.

Results: The majority of residents shopped at supermarkets (90\%), 64\% obtained food from neighborhood food pantries, and $41 \%$ reported difficulties finding fresh vegetables. Focus group data indicated desire for high-quality fresh produce at affordable prices and a large variety of options. OLs identified "having a proper distribution channel" for food from regional farms and "increasing community farms and gardens" as solutions to bring more regional foods into communities. Those 